Published in final edited form as:

Curr Opin Gastroenterol. 2012 March ; 28(2): 130-134. doi:10.1097/MOG.0b013e32834e7bc3.

\title{
Small intestinal ion transport
}

\author{
Fayez K. Ghishan ${ }^{a}$ and Pawel R. Kiela ${ }^{a, b}$ \\ aDepartment of Pediatrics, Steele Children's Research Center, University of Arizona Health \\ Science Center, Tucson, Arizona, USA

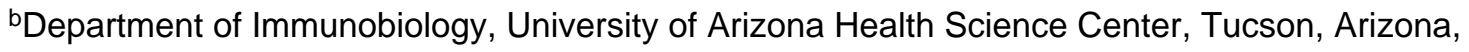 \\ USA
}

\section{Abstract}

Purpose of review-In this review, we focus on the recent (March 2010 to September 2011) advances in small intestinal ion transport, with particular emphasis on sodium, chloride, bicarbonate, and calcium transport mechanisms under physiological and pathophysiological conditions.

Recent findings-Knockout of NHERF1 and NHERF2 allowed translation of the data largely derived from the in-vitro models into a living organism. These studies also expand our knowledge about the complexity of intestinal transporter interactomes, define the role for scaffolding proteins in basal and regulated apical transport, and help identify potential targets for pharmacological approaches. We continue to accumulate novel information about the function and regulation of NHE3 (including its role in regulating paracellular $\mathrm{Ca}^{2+}$ flux), NHE8, as well as about the complexity of the intestinal $\mathrm{Cl}^{-}$and $\mathrm{HCO}_{3}{ }^{-}$transport in health and disease.

Summary-Thanks to the new genetically engineered mouse models, a significant progress has been made in our understanding of the role of NHERF proteins in regulation of intestinal $\mathrm{Na}^{+}$ absorption. Significant novel data on the coordinated function of bicarbonate, chloride, and sodium transporters contributes to our current views of the integrative physiology of the small intestinal electrolyte transport.

\section{Keywords}

anion exchange; bicarbonate secretion; chloride transport; diarrhea; $\mathrm{Na}^{+} / \mathrm{H}^{+}$exchange; PDZ proteins

\section{INTRODUCTION}

Secretion and absorption of electrolytes and fluid are two essential functions of the small intestinal epithelial cells. In healthy adult humans, the gastrointestinal tract is capable of

Copyright $\odot$ Lippincott Williams \& Wilkins. Unauthorized reproduction of this article is prohibited.

Correspondence to Fayez K. Ghishan, Department of Pediatrics, Steele Children's Research Center, University of Arizona Health Sciences Center, 1501 North Campbell Avenue, Tucson, AZ 85724, USA. Tel: +1 520626 5170; fax: +1 520626 4141; fghishan@peds.arizona.edu.

Conflicts of interest

There are no conflicts of interest. 
secreting 8-10 1 of fluid per day in addition to $1.5-21$ per day from ingested food. To reduce this volume to the typical ileocecal daily flow of 21 , small intestinal epithelium faces an enormous challenge. To accomplish this task, a large number of transport molecules act in a synergistic, additive, or antagonistic way together with an array of scaffolding and regulatory proteins. The net fluid movement across the gastrointestinal epithelium is primarily the result of active transport of $\mathrm{Na}^{+}, \mathrm{Cl}^{-}$, and $\mathrm{HCO}_{3}{ }^{-}$, among others. Disturbances of these coordinated transport events, either a result of genetic mutations, pathogenic infections, or dysregulation (e.g. during inflammation), often result in diarrhea when colonic capacity for fluid absorption is overwhelmed.

Every year, new discoveries help us understand the mechanism governing intestinal fluid and electrolyte absorption. They also unravel potential targets for therapeutic approaches which could limit fluid losses and the resulting morbidity and mortality worldwide. The topics covered range from studies on tissue distribution and cellular localization, transcriptional and posttranscriptional regulation, to pathophysiology of diarrhea.

In addition to the most recent review on small intestinal ion transport in Current Opinion in Gastroenterology by Venkatasubramanian et al. [1"'], several comprehensive reviews on these topics have recently been published. Transcriptional regulation of intestinal electrolyte transporters has been reviewed by Malakooti et al. [2"]. Regulation of the small intestinal electroneutral $\mathrm{NaCl}$ absorption was extensively covered by Kato and Romero [3"]. Certain aspects of duodenal bicarbonate secretion have been reviewed by Takeuchi et al. [4].

A significant number of articles discussed in this reviewed period have focused on the mechanisms of acute regulation of apical electrolyte transport and their dependence on PSD95, Drosophila homolog Disk-large, zona occludens 1 (ZO-1) (PDZ) adapter proteins. We will also review new data on the most recently described intestinal $\mathrm{Na}^{+} / \mathrm{H}^{+}$exchanger, NHE8, contribution of NHE3-mediated $\mathrm{Na}^{+}$flux to the paracellular $\mathrm{Ca}^{2+}$ transport, and cover some of the newest data on intestinal bicarbonate and chloride transport.

\section{NHERF PROTEINS IN NHE3-MEDIATED Na+ ABSORPTION}

The four NHERF $\left(\mathrm{Na}^{+} / \mathrm{H}^{+}\right.$exchanger regulatory factor) $\mathrm{PDZ}$ adapter proteins can bind to a variety of membrane transporters and receptors and modulate their membrane targeting, mobility, interaction with other proteins, and the formation of signaling complexes. Among the most prominent intestinal electrolyte transporters containing the PDZ-binding motifs enabling their interaction with NHERF1, NHERF2 (E3KARP), and NHERF3 (CAP70, PDZK1), are the cystic fibrosis transmembrane regulator (CFTR) anion channel and the $\mathrm{Na}^{+} / \mathrm{H}^{+}$exchanger NHE3.

Although much of the information on the role and function of the NHERF proteins has come from in-vitro studies and frequently from cell culture models with forced expression of the proteins, recent targeted knockouts of NHERF1 and NHERF2 provide the tools for verification of some of these findings. Murtazina et al. [5"'] have demonstrated that NHERF2-null knockout mice have altered ileal mucosal architecture, with shorter villi, deeper crypts, and decreased epithelial cell number. Contrary to another report from the same laboratory, which described the shRNA-mediated NHERF2 knockdown resulted in 
increased NHE3 activity in Caco-2/bbe cells [6], basal NHE3 activity was reduced in NHERF2 ${ }^{-/-}$mice [5"']. This was accompanied by reduced proportion of NHE3 in the apical domain, and an increase in its intracellular pool, without changes in total NHE3 protein or mRNA expression. However, both the in-vitro and the in-vivo approaches consistently demonstrated that NHERF2 was necessary for cGMP-dependent protein kinase (cGK)-II and $\mathrm{Ca}^{2+}$-dependent inhibition of NHE3. The difference in the effects of NHERF2 knockdown or deficiency in cells vs. intact ileum was interpreted by the authors by NHERF2 playing a critical role in maintaining a mobile subapical pool of NHE3 which was not accessible to apical surface biotinylation and which moved more apically when NHERF2 was reduced [5"',6,7"']. These studies along with another report from Chen et al. [8] all strongly suggest that the primary role of NHERF2 is to maintain mobile sub-apical fraction of NHE3 and is critical in acute regulation of NHE3 activity relying on rapid mobilization to or away from the brush border membrane.

This function of NHERF2 was also exemplified by another recent study by Lin et al. [7"']. Sodium-dependent glucose transport via SGLT1 is the foundation of effective oral

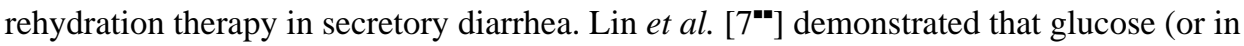
this case nonmetabolized a-methyl-D-glucose) not only activates small intestinal NHE3, but also that it does so in a mechanism dependent on Akt kinase and NHERF2 (but not NHERF1). In Caco-2 cells, this glucose-mediated NHE3 stimulation was also NHREF2 dependent, associated with increased surface expression of NHE3, and with a dissociation of the NHERF2-NHE3 complex, increase in the mobile fraction of NHE3, and increase in association of NHE3 with ezrin. Most importantly, glucose fully reversed cholera toxininduced inhibition of NHE3 activity, thus adding a new dimension to the protective mechanisms of oral rehydration in acute watery diarrhea. Interestingly, another study by Coon et al. [9], which also explored the interaction between SGLT1 and NHE3, demonstrated that expression of these two transporters is linked to their function. This was demonstrated in vitro in IEC-18 cells transfected with either NHE3 or SGLT1 siRNA. Expression and function of the two carriers were reciprocally regulated, that is, knockdown of SGLT1 increased NHE3 expression and activity and vice versa [9].

Lysophosphatidic acid (LPA), a small, bioactive glycerophospholipid, plays an important role in regulating intestinal electrolyte transport through stimulation of NHE3 activity, inhibition of CFTR-dependent $\mathrm{Cl}^{-}$secretion, and stimulation of $\mathrm{Cl}^{-} / \mathrm{OH}^{-}$exchange. Yoo et al. $\left[10^{-"}\right]$ demonstrated that activation of NHE3 by LPA is mediated via LPA 5 receptor and results in obligatory activation of the apically expressed EGFR pool. EGFR activation following LPA treatment resulted in activation of the MEK-Erk kinase pathway, activation of RhoA and Rho-associated kinase (ROCK), and downstream activation of proline-rich tyrosine kinase 2 (Pyk2). Knockdown of Pyk2 blocked NHE3 activation by LPA.

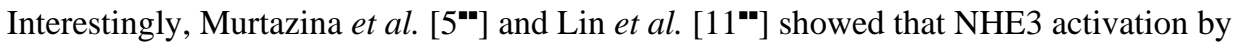
luminal LPA was abolished in the absence of NHERF2. The relationship between the signaling pathway described by Yoo et al. [10"'], and Pyk2 in particular, with the NHERF2/ NHE3 complex is yet to be elucidated. These studies, however, point to a potential use of $\mathrm{LPA}_{5}$ agonists for the treatment of diarrheal disorders. 
A larger scale approach to the effects of NHERF1 and NHERF2 deficiency has been published by Donowitz et al. [12,13]. In these two studies, jejunal brush borders were purified from NHERF1 ${ }^{-/-}$, NHERF2 ${ }^{-/-}$, or wild-type mice and associated proteins characterized using mass spectrometry. This approach identified alterations in multiple transport proteins, signaling molecules, cytoskeletal proteins, tight junctional and adherens junction proteins, and proteins involved in metabolism and proliferations, thus highlighting the importance of NHERF1 and NHERF2 in epithelial biology. However, NHE3, a subject of many other detailed studies, has not been identified, thus raising a possibility that this experimental approach may have missed some other key players as well. These two studies may, however, provide a valuable hypothesis-generating tool to investigate other, previously unappreciated roles for these two scaffolding molecules.

\section{NHE8}

NHE8 is the newest member of the sodium/hydrogen exchanger family. NHE8 has been proposed to regulate medialltrans-Golgi $\mathrm{pH}$, and more recently to regulate the morphology and function of the late endosomal compartment [14]. Interestingly, this NHE8 function could not be attributed to its $\mathrm{Na}^{+} / \mathrm{H}^{+}$exchange activity, as its depletion did not cause distinguishable changes in the $\mathrm{pH}$ within the dense multivesicular bodies. In the small intestine, a significant fraction of NHE8 protein is located at the apical membrane, where it is believed to play an important role in electrolyte absorption during early stages of postnatal development. Characterization of the intestinal expression of NHE8 raised a question whether mutation in this gene could underlie the pathogenesis of congenital sodium diarrhea, a rare genetic disorder characterized by defective $\mathrm{Na}^{+} / \mathrm{H}^{+}$exchange, which has been shown to remit in some patients when they reach the first year of life. Baum et al. [15] sequenced the immediate promoter region, exons and intron/exon boundaries of the NHE8 gene from five patients diagnosed with congenital sodium diarrhea, but failed to identify any disease-causing base changes. However, NHE8 remains an attractive target for pharmacological strategies aimed at prevention and treatment of diarrhea. Octreotide, a somatostatin analog, which has been used off-label for the treatment of severe, refractory diarrhea, was shown to selectively increase expression of NHE8 in the mouse small intestine and in Caco-2 cells [16"]. This increase was mediated by SSTR2 receptor and required p38 MAPK activation. High early postnatal intestinal expression of NHE8 has also been proposed to be the basis of higher survival of NHE2/NHE3 double knockout mice before weaning, and a declining survival at later stages [17"]. This protection was particularly apparent in female mice, which had higher expression of NHE3 after weaning than male mice. Consistently, in Caco-2 cells, testosterone, but not estrogen, reduced NHE8 expression at the transcriptional level, indicating a sex-specific compensatory role of NHE8 in compounded NHE2/NHE3 deficiency [17"]. Another study by Xu et al. [18] showed that epidermal growth factor (EGF), known for its stimulatory effects on intestinal maturation and NHE2 activity, downregulated intestinal NHE8 expression by modulating Sp3 binding to the proximal promoter of the NHE8 gene, thus suggesting an important role of EGF in modulating the expression and activity of this $\mathrm{Na}^{+} / \mathrm{H}^{+}$exchanger. 


\section{CONTRIBUTION OF NHE3 TO INTESTINAL PARACELLULAR $\mathrm{Ca}^{2+}$ TRANSPORT}

Depending on the segment and the luminal $\mathrm{Ca}^{2+}$ concentration, intestinal absorption occurs via a weakly regulated paracellular route (dominant under normal dietary calcium levels), or by a highly regulated, TRPV6-mediated and $1,25(\mathrm{OH})_{2}$ vitamin $\mathrm{D}_{3}$-sensitive transcellular pathway. It has been hypothesized that similar to renal proximal tubules, where $\mathrm{Ca}^{2+}$ is reabsorbed via a passive paracellular process with the active transcellular flux of $\mathrm{Na}^{+}$ serving as the driving force, passive $\mathrm{Ca}^{2+}$ transport in the intestine is similarly regulated. Two potential mechanisms were suggested to explain how the active transcellular $\mathrm{Na}^{+}$flux mediates passive paracellular $\mathrm{Ca}^{2+}$ absorption: removal of water concentrates luminal $\mathrm{Ca}^{2+}$ thereby generating a chemical gradient and water flux drives $\mathrm{Ca}^{2+}$ flux via the 'solvent drag' mechanism. A recent report by Pan et al. [19"] showed that NHE3 activity provides the major driving force for paracellular $\mathrm{Ca}^{2+}$ transport. $\mathrm{NHE}^{-1-}$ mice, despite the elevated levels of $1,25(\mathrm{OH})_{2}$ vitamin $\mathrm{D}_{3}$, displayed decreased renal and intestinal $\mathrm{Ca}^{2+}$ uptake, increased fractional $\mathrm{Ca}^{2+}$ excretion, and lower mineral bone density [19"]. The authors also linked the decreased intestinal $\mathrm{Ca}^{2+}$ transport to decreased jejunal expression of claudin-2, known for its ability to form $\mathrm{Ca}^{2+}$-permeable paracellular pores. This observation is, however, in contrast with our earlier report which showed by microarray and real-time RTPCR analysis that $\mathrm{NHE}^{-/-}$overexpress claudin-2 in the small intestinal epithelium [20]. Although not without conflicting data, this manuscript clearly indicated the role for NHE3 as the major driving force in intestinal and renal paracellular $\mathrm{Ca}^{2+}$ flux.

\section{INTESTINAL Cl- AND HCO3- TRANSPORT}

Three anion/ $\mathrm{HCO}_{3}{ }^{-}$exchangers, SLC26A3 (DRA: downregulated in adenoma), SLC26A6 (putative anion transporter 1, PAT1), and SLC4A9 (AE4), provide the major pathways for duodenal bicarbonate secretion, a critical nonstructural protection mechanism against the acidic gastric output, and changes in luminal $\mathrm{pH}$, which could adversely affect mucin viscosity and bacterial binding, and against mucosal ulcerations. Mutations within the SLC26A3 gene (responsible for epithelial $\mathrm{Cl}^{-} / \mathrm{HCO}_{3}{ }^{-}$and $\mathrm{Cl}^{-} / \mathrm{OH}^{-}$exchange) are responsible for congenital chloride diarrhea (recently reviewed by Wedenoja et al. [21]). Moreover, changes in ileocolonic lumen $\mathrm{pH}$ in inflammatory bowel disease patients have been attributed to reduced $\mathrm{HCO}_{3}{ }^{-}$secretion, a hypothesis recently confirmed in a murine model of ileitis (TNF $\triangle \mathrm{ARE}$ mice) [22"]. In this latter study, suppression of epithelial $\mathrm{HCO}_{3}{ }^{-}$ secretory rate in both ileum and mid-colon was accompanied by a strong reduction in DRA mRNA and protein expression without concomitant changes in the expression of CFTR, NHE3, basolateral $\mathrm{NBC}\left(\mathrm{Na}^{+} / \mathrm{HCO}_{3}{ }^{-}\right.$co-transporter), or epithelial sodium channel (ENaC). Although fluid absorption in the ileum was significantly impaired, the colon provided sufficient compensation to prevent the development of overt diarrhea. Interestingly, in another study, Raheja et al. [23] demonstrated that a probiotic Lactobacillus acidophilus administered to mice increases colonic DRA (but not PAT1) expression, a phenomenon attributed to a transcriptional activation of the SLC26A3 gene promoter. However, no such effect was observed in the jejunum or ileum of mice colonized with L. acidophilus. This finding may be related to preferential colonization of the large bowel by L. acidophilus or by 
differences in regulatory pathways governing the expression of DRA along the gastrointestinal tract (lower in the jejunum and ileum than colon).

CFTR has been postulated to function not only as a chloride channel, but also as a key regulator of duodenal bicarbonate secretion. Using a series of elegant studies with knockout mice and specific inhibitors, Singh et al. [24"'] showed that inhibition or ablation of NHE3 activity led to elevated or 'unmasked' $\mathrm{HCO}_{3}{ }^{-}$secretion mediated by DRA and PAT1. However, inhibition or deletion of CFTR abolished the effect of NHE3 inhibitor on duodenal bicarbonate secretion, thus providing novel evidence that CFTR activity (and not just its presence) is a prerequisite for duodenal $\mathrm{HCO}_{3}{ }^{-}$secretion. This was not the case for SLC26-mediated duodenal fluid absorption which required coupling with NHE3 activity [24"']. The authors concluded with a hypothesis that PAT1 and DRA require NHE3mediated proton recycling in order to operate in the $\mathrm{Cl}^{-}$absorptive mode, as well as $\mathrm{Cl}^{-}$exit via CFTR to operate in the $\mathrm{HCO}_{3}{ }^{-}$secretory mode.

Another aspect of diarrheal diseases - enterotoxin-induced, CFTR-mediated $\mathrm{Cl}^{-}$secretion was investigated by Collaco et al. [25]. In the rat jejunum and Caco-2 bbe cells, the cGMP agonist STa induced exocytic insertion of CTFR into the apical membrane along with syntaxin 3. Syntaxin 3 is a protein involved in apical recycling and in biosynthetic traffic from the trans-Golgi network (TGN) to the apical surface of epithelial cells, and a member of the N-ethylmaleimide sensitive factor attachment protein (SNARE) family. shRNAmediated knockdown of syntaxin 3 in Caco- 2 cells reduced the effect of cAMP on surface expression of CFTR. This observation suggests that in addition to pharmacological approaches targeting CFTR channels on the enterocyte surface, exocytosis-based mechanisms responsible for the increase in surface CFTR expression can also potentially prove to be vital targets for the therapy of enterotoxin-induced diarrhea as well as of cystic fibrosis. A review expanding upon the role of the SNARE family proteins in the regulation of CFTR trafficking has been recently published by Tang et al. [26].

\section{CONCLUSION}

Genetically engineered mouse models lacking some of the key genes encoding transporters and their adapter proteins continue to provide important clues into intestinal transport processes, and help verify previous data obtained from reductionist in-vitro models. Some of the recently published work identifies potential mechanisms in the pathogenesis of diarrhea and unveils potential targets for future pharmacological approaches. Novel information on the interdependent functions of multiple transporters, and transport processes, for example, epithelial $\mathrm{Na}^{+}$and $\mathrm{Ca}^{2+}$ flux, or intestinal chloride and bicarbonate secretion, continue adding to an already quite complex picture of the integrative physiology of the small intestinal electrolyte transport.

\section{Acknowledgments}

None.

This work was supported by grants from the National Institute of Diabetes and Digestive and Kidney Diseases [2R01 DK041274 (to F. K. Ghishan) and 5R01 DK067286-07 (to P.R. Kiela)]. 


\section{REFERENCES AND RECOMMENDED READING}

Papers of particular interest, published within the annual period of review, have been highlighted as:

- of special interest

- of outstanding interest

Additional references related to this topic can also be found in the Current World Literature section in this issue (p. 182).

1-. Venkatasubramanian J, Ao M, Rao MC. Ion transport in the small intestine. Curr Opin Gastroenterol. 2010; 26:123-128. This 2010 review on small intestinal ion transport focuses on the recent advances in the field with particular emphasis of the pathophysiology of intestinal disorders. [PubMed: 20010100]

2-. Malakooti J, Saksena S, Gill RK, Dudeja PK. Transcriptional regulation of the intestinal luminal $\mathrm{Na}$ and $\mathrm{Cl}$ transporters. Biochem J. 2011; 435:313-325. This recent review provides a detailed analysis of the core promoters of NHE2, NHE3, DRA, and PAT-1 and outlines the transcription factors involved in their basal regulation and in response to physiological and pathophysiological stimuli. [PubMed: 21726200]

3-. Kato A, Romero MF. Regulation of electroneutral $\mathrm{NaCl}$ absorption by the small intestine. Annu Rev Physiol. 2011; 73:261-281. In this review, Kato and Romero provide a comprehensive review of nutrient-coupled $\mathrm{Na}^{+}$absorption, electroneutral $\mathrm{NaCl}$ absorption, electrogenic $\mathrm{Cl}^{-}$ secretion, and electrogenic $\mathrm{Na}^{+}$absorption in the mammalian intestine. [PubMed: 21054167]

4. Takeuchi K, Kita K, Hayashi S, Aihara E. Regulatory mechanism of duodenal bicarbonate secretion: Roles of endogenous prostaglandins and nitric oxide. Pharmacol Therapeut. 2011; 130:59-70.

5m. Murtazina R, Kovbasnjuk O, Chen TE, et al. NHERF2 is necessary for basal activity, second messenger inhibition, and LPA stimulation of NHE3 in mouse distal ileum. Am J Physiol Cell Physiol. 2011; 301:C126-C136. This study provides description of morphological and functional changes in the small intestinal mucosa of NHERF2 ${ }^{-/}$mice and verifies in vivo the role of this adapter protein in basal and regulated activity of NHE3 as well as its intracellular localization. [PubMed: 21430287]

6. Sarker R, Valkhoff VE, Zachos NC, et al. NHERF1 and NHERF2 are necessary for multiple but usually separate aspects of basal and acute regulation of NHE3 activity. Am J Physiol Cell Physiol. 2011; 300:C771-C782. [PubMed: 21191106]

7-. Lin R, Murtazina R, Cha B, et al. D-glucose acts via sodium/glucose cotransporter 1 to increase NHE3 in mouse jejunal brush border by a $\mathrm{Na}+\mathrm{H}+$ exchange regulatory factor 2-dependent process. Gastroenterology. 2011; 140:560-571. In this report, the authors characterize the effects of glucose on NHE3-mediated sodium absorption, its NHERF2 dependency. This finding is of great significance for our current understanding of the effectiveness and mechanisms of oral rehydration in secretory diarrhea. [PubMed: 20977906]

8. Chen M, Sultan A, Cinar A, et al. Loss of PDZ-adaptor protein NHERF2 affects membrane localization and cGMP- and [Ca2+]-but not cAMP-dependent regulation of $\mathrm{Na}^{+} / \mathrm{H}^{+}$exchanger 3 in murine intestine. J Physiol. 2010; 588:5049-5063. [PubMed: 20962002]

9. Coon S, Kekuda R, Saha P, Sundaram U. Reciprocal regulation of the primary sodium absorptive pathways in rat intestinal epithelial cells. Am J Physiol Cell Physiol. 2011; 300:C496-505. [PubMed: 21148403]

10-r. Yoo BK, He P, Lee SJ, Yun CC. Lysophosphatidic acid 5 receptor induces activation of $\mathrm{Na}^{+} / \mathrm{H}^{+}$ exchanger 3 via apical epidermal growth factor receptor in intestinal epithelial cells. Am $\mathbf{J}$ Physiol Cell Physiol. 2011; 301:C1008-C1016. This study describes the mechanism of LPAmediated induction of NHE3 activity, the role of LPA5 receptor, and the contribution of EGFRmediated signaling and Pyk2 kinase. [PubMed: 21832242]

11-r. Lin S, Yeruva S, He P, et al. Lysophosphatidic acid stimulates the intestinal brush border $\mathrm{Na}(+) /$ $\mathrm{H}(+)$ exchanger 3 and fluid absorption via LPA(5) and NHERF2. Gastroenterology. 2010; 
138:649-658. The data provided in this manuscript confirm and expand upon the findings of Lin et al. [11"] to provide evidence for the critical involvement of NHERF2 in LPA-stimulated NHE3 activity. [PubMed: 19800338]

12. Donowitz M, Singh S, Singh P, et al. Alterations in the proteome of the NHERF1 knockout mouse jejunal brush border membrane vesicles. Physiol Genomics. 2010; 42A:200-210. [PubMed: 20736413]

13. Donowitz M, Singh S, Singh P, et al. Alterations in the proteome of the NHERF2 knockout mouse jejunal brush border membrane vesicles. Physiol Genomics. 2011; 43:674-684. [PubMed: 21427361]

14. Lawrence SP, Bright NA, Luzio JP, Bowers K. The sodium/proton exchanger NHE8 regulates late endosomal morphology and function. Mol Biol Cell. 2010; 21:3540-3551. [PubMed: 20719963]

15. Baum M, Martin MG, Booth IW, et al. Nucleotide sequence of the $\mathrm{Na}^{+} / \mathrm{H}^{+}$exchanger-8 (NHE8) in patients with congenital sodium diarrhea. J Pediatr Gastroenterol Nutr. 2011; 53:474-477. [PubMed: 21666503]

16-. Wang C, Xu H, Chen H, et al. Somatostatin stimulates intestinal NHE8 expression via p38 MAPK pathway. Am J Physiol Cell Physiol. 2011; 300:C375-C382. The study described the effects and mechanisms of action of octreotide, a somatostatin analog used off-label for the treatment of severe, refractory diarrhea, as selectively increasing the expression of NHE8 in the mouse small intestine and in Caco-2 cells. [PubMed: 21106692]

17m. Xu H, Li J, Chen R, et al. NHE2X3 DKO mice exhibit gender-specific NHE8 compensation. Am J Physiol Gastrointest Liver Physiol. 2011; 300:G647-G653. This article addresses the contribution of NHE8 to small intestinal $\mathrm{Na}^{+} / \mathrm{H}^{+}$exchange utilizing NHE2/NHE3 double knockout mice, characterizes sex-specific, NHE8-mediated compensation in the absence of the two major apical exchanges, and regulation of NHE8 expression by testosterone. [PubMed: 21252044]

18. Xu H, Zhang B, Li J, et al. Epidermal growth factor inhibits intestinal NHE8 expression via reducing its basal transcription. Am J Physiol Cell Physiol. 2010; 299:C51-C57. [PubMed: 20375273]

19-. Pan W, Borovac J, Spicer Z, et al. The epithelial sodium-proton exchanger, Nhe3, is necessary for renal and intestinal calcium (Re)absorption. Am J Physiol Renal Physiol. 2011 [Epub ahead of print]. In this report, Pan et al. demonstrate decreased intestinal and renal calcium absorption in NHE3-deficient mice, which coincides with decreased bone mineral density. NHE3 activity is postulated to provide the main driving force for paracellular $\mathrm{Ca}^{2+}$ flux.

20. Kiela PR, Laubitz D, Larmonier CB, et al. Changes in mucosal homeostasis predispose NHE3 knockout mice to increased susceptibility to DSS-induced epithelial injury. Gastroenterology. 2009; 137:965-975. 975.e1-975.e10. [PubMed: 19450596]

21. Wedenoja S, Pekansaari E, Hoglund P, et al. Update on SLC26A3 mutations in congenital chloride diarrhea. Hum Mutat. 2011; 32:715-722. [PubMed: 21394828]

22. Xiao F, Juric M, Li J, et al. Loss of downregulated in adenoma (DRA) impairs mucosal HCO(3) (-) secretion in murine ileocolonic inflammation. Inflamm Bowel Dis. 2011 [Epub ahead of print]. Xiao et al. describe the suppression of epithelial bicarbonate secretory rate in a model of ileocecal inflammation (TNF $\triangle$ ARE mice). This inhibition was accompanied by significantly decreased expression of DRA. The authors postulate that this may contribute to alterations in surface $\mathrm{pH}$, intestinal flora, and mucus barrier properties.

23. Raheja G, Singh V, Ma K, et al. Lactobacillus acidophilus stimulates the expression of SLC26A3 via a transcriptional mechanism. Am J Physiol Gastrointest Liver Physiol. 2010; 298:G395-G401. [PubMed: 20044511]

24m. Singh AK, Riederer B, Chen M, et al. The switch of intestinal Slc26 exchangers from anion absorptive to HCOFormula secretory mode is dependent on CFTR anion channel function. Am J Physiol Cell Physiol. 2010; 298:C1057-C1065. Singh et al. studied the relationship between NHE3, CFTR, and the activities of the two $\mathrm{Cl}^{-} / \mathrm{HCO}_{3}{ }^{-}$exchangers, DRA and PAT1. Their data suggest that DRA and PAT1 need proton recycling via NHE3 to operate in the $\mathrm{Cl}^{-}$absorptive mode and $\mathrm{Cl}^{-}$exit via CFTR to operate in the $\mathrm{HCO}_{3}{ }^{-}$secretory mode. [PubMed: 20164375] 
25. Collaco A, Marathe J, Kohnke H, et al. Syntaxin 3 is necessary for cAMP-and cGMP-regulated exocytosis of CFTR: implications for enterotoxigenic diarrhea. Am J Physiol Cell Physiol. 2010; 299:C1450-C1460. [PubMed: 20844248]

26. Tang BL, Gee HY, Lee MG. The cystic fibrosis transmembrane conductance regulator's expanding SNARE interactome. Traffic. 2011; 12:364-371. [PubMed: 21223469] 


\section{KEY POINTS}

- $\quad$ NHERF2 serves a key role in the maintenance of a regulated pool of NHE3 protein and is critical in dynamic responses in intestinal $\mathrm{Na}^{+} / \mathrm{H}^{+}$exchange to physiological and pharmacological stimuli.

- Activation of NHE3 by glucose may be an important component of the protection offered by oral rehydration solution in secretory diarrhea.

- $\quad$ NHE8 emerges as a contributor to intestinal $\mathrm{Na}^{+} / \mathrm{H}^{+}$exchange.

- Epithelial bicarbonate secretion, a critical nonstructural protection mechanism, is reduced in intestinal inflammation but can be enhanced by selected probiotics.

- Intestinal transporters do not work in isolation, but rather form functional networks based on functional and physical interactions, and common regulatory pathways. 